nadores, que foram devidamente treinados e realizaram um exercício de calibração antes da análise. Passadas 2 semanas foi feita uma nova análise em 30 scans escolhidos aleatoriamente, de modo a permitir a análise do erro intra-examinador. O Coeficiente de Kappa foi utilizado para avaliar o erro intra e inter examinador. O Coeficiente de Correlação de Spearman e - Coeficiente de Correlação Intraclasses foram utilizados para avaliar a correlação entre as 2 escalas. O tratamento estatístico dos dados foi realizado com recurso ao software SPSS ${ }^{\circledR}$. Considerou-se um nível de significância de 5\%. Resultados: Os valores do Coeficiente de Kappa foram maiores que 0,85, tanto na avaliação do erro inter como do erro intra observador, para ambas as escalas. Os valores do Coeficiente de Correlação de Spearman $(0,838)$ e do Coeficiente de Correlação Intraclasses $(0,883)$ mostram a existência de uma forte correlação entre as 2 escalas. Conclusões: Os resultados deste estudo demonstram que as escalas de BOKA e Angelieri et al. são ambas reprodutíveis e que têm uma forte correlação entre si, o que leva à conclusão de que a escala BOKA parece ser efetiva na avaliação do grau de maturação da sutura palatina.

http://doi.org/10.24873/j.rpemd.2019.12.552

\section{\#091 Avaliação da largura cortical mandibular em TCs de pessoas com doença falciforme}

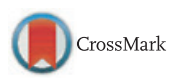

Renata da Paz Leal Pereira*, Juliana Jorge Garcia, Emi Suzane de Abreu Dias, Viviane de Almeida Sarmento, Heloísa Laís Rosario dos Santos, Viviane de Sousa Moreira Almeida

Universidade Federal da Bahia

Objetivos: O objetivo do presente estudo foi avaliar a largura da cortical mandibular (LCM) em indivíduos portadores de doença falciforme. Materiais e métodos: Setenta e cinco reconstruções panorâmicas de tomografias computadorizadas de pacientes portadores de doença falciforme foram coletadas para a análise da Largura da Cortical Mandibular na região mentual (LCM). A análise foi realizada por dois avaliadores. Em cada exame, o LCM foi medido dos lados da mandíbula, duas vezes por um mesmo avaliador, com intervalo de no mínimo sete dias entre as avaliações. Para avaliação do LCM, foi medido o valor de ' $C$ ' que corresponde a altura da cortical mandibular abaixo do forame mentual. Os dados foram registrados em uma tabela e enviados para posterior análise estatística. Resultados: Inicialmente foi calculada a média das duas medidas realizadas em cada lado da mandíbula pelo avaliador e, em seguida, foi calculada a média do LCM entre os dois lados da mandíbula de cada paciente. O valor de LCM foi considerado normal quando igual ou maior a $4 \mathrm{~mm}$. Os resultados revelaram uma média de LCM de $4,1 \mathrm{~mm}$ (com desvio-padrão de $0,7 \mathrm{~mm}$ ). Vinte e sete pacientes $(36,5 \%)$ apresentaram valores de LCM diminuídos e 47 tiveram valores considerados normais (63,5\% da amostra) Discussão: Os resultados demonstraram que na amostra avaliada uma grande parte dos pacientes já apresentavam diminuição da massa óssea mandibular detectada por este índice. Radiografias odontológicas podem revelar o efeito que a doença falciforme tem sobre o metabolismo ósseo, evidenciando perdas ósseas e más oclusões decorrentes da hiperplasia medular compensatória. Conclusões: A largura da cortical mandibular pode ser utilizada para avaliar osteopenia resultante de diversas condições sistêmicas e nos Indivíduos com doença falciforme este índice pode demonstrar a presença de alterações ósseas. http://doi.org/10.24873/j.rpemd.2019.12.553

\section{\#92 Influência da anestesia: Subperióstea} Vs Loco-regional - Ensaio Clínico Aleatorizado

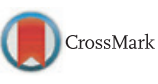

Ana Rita Jorge Reis*, André Chen, Helena Francisco, Joana Fialho, João Caramês

Faculdade de Medicina Dentária da Universidade de Lisboa, Escola Superior de Tecnologia e Gestão de Viseu; Centro de Estudos em Educação, Tecnologia e Saúde

Objetivos: Quando é necessário realizar exodontia de 1os ou 2os molares mandibulares a escolha da técnica anestésica da maioria dos profissionais de saúde oral irá recair sobre o bloqueio loco-regional, no entanto, com o aparecimento da articaína pode ser possível alcançar uma anestesia eficaz pela técnica subperióstea. O objetivo deste estudo é comparar a eficácia anestésica do Cloridrato de articaína a 4\% com epinefrina a 1:200 000 na exodontia de 1os e 2os molares mandibulares quando utilizada anestesia subperióstea vs. anestesia loco-regional. Materiais e métodos: Ensaio clínico aleatorizado com 15 pacientes, que se dirigiram à clínica universitária da Faculdade de Medicina Dentária da Universidade de Lisboa, para a extração de 1 os ou 20 molares mandibulares. Após aleatorização os pacientes receberam anestesia loco-regional ou anestesia infiltrativa de acordo com o grupo atribuído. De forma a determinar a eficácia anestésica alcançada para cada uma das técnicas, foi registada numa escala VAS (Visual Analogue Scale) a dor sentida antes, durante e após o procedimento e a dor durante a anestesia. Foi registada pelo clínico a quantidade de anestésico utilizado. Resultados: Não foram encontradas diferenças estatisticamente significativas $(p>0,05)$ quando se compara a dor sentida antes, durante e após o procedimento e a dor durante a anestesia. Foram verificadas diferenças estatisticamente significativas $(p<0,05)$ entre a quantidade de anestésico utilizado pelas duas técnicas. Conclusões: Os resultados deste estudo sugerem que não existe diferença na eficácia anestésica do Cloridrato de articaína a 4\% com epinefrina a 1:200 000 na exodontia de 1 os e 20 s molares mandibulares quando utilizada anestesia subperióstea vs. anestesia loco-regional. http://doi.org/10.24873/j.rpemd.2019.12.554

\section{\#093 Endocrown Vs. Restauração parcial cerâmica com pino de fibra - Estudo clínico randomizado \\ CrossMark}

Susana Morimoto*, Reinaldo Macedo Fraga, Tamara Kerber Tedesco, Mutlu Özcan, Maria Stella Nunes A Moreira, Daniela Prócida Raggio

Universidade de São Paulo- FOUSP, University of Zürich, Switzerland, UniversidadeIbirapuera, São Paulo, Brasil, Universidade Ibirapuera, São Paulo, Brasil

Objetivos: Comparar o tempo operatório, desconforto e satisfação de pacientes restaurados com endocrowns cerâmi- 
cas ou com restaurações parciais (inlays/ onlays/ overlays) cerâmicas com pino de fibra e preenchimento de resina composta (Rest Parcial pino). Materiais e métodos: Um ensaio clínico controlado randomizado duplo-cego foi conduzido por apenas 1 operador, dentro dos critérios de elegibilidade, randomização e sigilo de alocação. Os critérios de elegibilidade foram: 1) Molares ou pré-molares com tratamento endodôntico e pelo menos uma parede de $3 \mathrm{~mm}$ de espessura; 2) Presença de dentes antagonistas; 3) Margens gengivais do preparo que permitiram adequada moldagem; e 4) Boa higiene bucal. Foram distribuídos aleatoriamente 20 participantes nos dois grupos $(n=40)$, sendo que apenas 1 dente foi incluído por paciente. As peças foram fresadas em IPS e-max CAD, maquiadas e cimentadas. O tempo foi cronometrado em cada sessão, o desconforto foi mensurado por meio da escala VAS e a satisfação foi medida por meio de escala numérica. Análise de regressão de Poisson e análise de variância de medidas repetidas foram realizadas com nível de significância de 5\%. No grupo Rest Parcial pino, $90 \%$ dos participantes relataram pouco ou nenhum desconforto, enquanto 82,5\% do grupo Endocrown respondeu de maneira semelhante. Não houve associação entre as variáveis explicativas (idade, sexo, dente, tipo de tratamento restaurador, número de superfícies tratadas, presença de lesão endodôntica e tempo operatório) e o desconforto relatado pelos pacientes. O nível de satisfação foi de $100 \%$. O tempo operatório no grupo Endocrown $(129,7 \mathrm{~min} \pm 29,78)$ foi semelhante ao do grupo Rest. parcial Pino $(134,1 \mathrm{~min} \pm 29,64)$. Resultados: No grupo Rest Parcial pino, $90 \%$ dos pacientes relataram pouco ou nenhum desconforto, enquanto $82,5 \%$ do grupo Endocrown respondeu de maneira semelhante. Não houve associação entre as variáveis explicativas (idade, sexo, dente, tipo de tratamento restaurador, número de superfícies tratadas, presença de lesão endodôntica e tempo operatório) e o desconforto relatado pelos pacientes. O nível de satisfação foi de $100 \%$. O tempo operatório no grupo Endocrown (129,7 $\min \pm 29,78)$ foi semelhante ao do grupo Rest. parcial Pino $(134,1 \mathrm{~min} \pm 29,64)$. Conclusões: Endocrowns e Rest Parcial pino possuem alto nível de satisfação, baixo desconforto e tempo operatório similar, contudo exigem menos passos clínicos e menor custo final, portanto, as endocrowns são excelentes alternativas para as Rest Parcial pino.

http://doi.org/10.24873/j.rpemd.2019.12.555

\section{\#094 Efetividade de três produtos de branqueamento dentário: ensaio clínico aleatorizado}

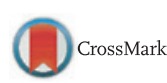

Ruben Pereira*, João Silveira, Susana Dias, Ana Beatriz Cardoso, António Mata, Duarte Marques

Faculdade de Medicina Dentária da Universidade de Lisboa

Objetivos: Avaliar a efetividade clínica de três produtos de branqueamento dentário com diferentes formulações. Materiais e métodos: Foi realizado um ensaio clínico aleatorizado com três grupos paralelos. Foram selecionados trinta pacientes por critérios de inclusão/exclusão, previamente definidos, e foram alocados a um dos três grupos de estudo: $6 \%$ peróxido de hidrogénio em consultório (VivaStyle ${ }^{\circledR}$ Paint On Plus, Ivo-
clarVivadent, Liechtenstein); 16\% peróxido de carbamida em ambulatório com moldeira individual (Opalescence ${ }^{\circledR} \mathrm{PF}$, Ultradent, EUA); 6\% peróxido de hidrogénio em ambulatório com moldeira adaptável (Opalescence ${ }^{\circledR}$ GO, Ultradent, EUA). Todos os voluntários realizaram uma consulta de profilaxia dentária, previamente ao início do tratamento, e a cor dentária dos 12 dentes anteriores de cada voluntário foi registada com um espectrofotómetro (SpectroShade, MHT Optic Research AG, Suiça). Foi realizado um novo registo da cor dentária a meio do tratamento e no final de 12 aplicações (10 minutos cada; 6 por sessão) de VivaStyle ${ }^{\circledR}$ Paint On Plus, 14 aplicações (4 a 6 horas cada) de Opalescence ${ }^{\circledR}$ PF ou 10 aplicações (60 a 90 minutos cada) de Opalescence ${ }^{\circledR} \mathrm{GO}$, segundo instruções do fabricante. Os resultados encontram-se expressos como média e desvio padrão de valores CIE L*a*b* e $\triangle E$. O teste t de Student, one-way ANOVA e Tukey post-hoc foram utilizados conforme apropriado com um nível de significância de $\alpha=0,05$. Resultados: Os valores de $\triangle \mathrm{E}$ para os dentes incisivos e caninos foram de 4,93 $\pm 1,84$ e 7,19 $\pm 1,75$ (VivaStyle ${ }^{\circledR}$ Paint On Plus), 6,84 $\pm 2,10$ e $11,53 \pm 2,84$ (Opalescence ${ }^{\circledR}$ PF) e 3,82 $\pm 2,04$ e 5,97 $\pm 2,35$ (Opalescence $^{\circledR} \mathrm{GO}$ ), respetivamente. Em todos os grupos, os valores CIE L*a*b* obtiveram diferenças estatisticamente significativas entre o início e fim do tratamento $(\mathrm{P}<0,01)$, no entanto na comparação entre grupos apenas estatisticamente significativas $(\mathrm{P}<0,01)$ para o Opalescence ${ }^{\circledR} \mathrm{PF}$ no grupo dos caninos. Conclusões: Todos os produtos demonstraram efeitos clínicos, com maior efetividade no grupo Opalescence ${ }^{\circledR}$ PF. O VivaStyle ${ }^{\circledR}$ Paint On Plus demonstra resultados promissores como técnica a utilizar em consultório, com um menor tempo de aplicação (2x60 minutos) quando comparado com as técnicas de ambulatório. Preconiza-se futuramente, o aumento da amostra em estudo com base na variabilidade obtida, bem como a avaliação da qualidade de vida dos pacientes durante o tratamento. http://doi.org/10.24873/j.rpemd.2019.12.556

\#095 Avaliação da saúde oral e sua relação com o SalivaPrint - Variação com idade e género

Marta André*, Eduardo Esteves, Bruna Correia, Mónica Fernandes, Nuno Rosa

Universidade Católica Portuguesa - Instituto de Ciências da Saúde

Objetivos: A saliva é atualmente utilizada para diagnóstico precoce e monitorização de patologias, tanto orais como sistémicas. No entanto, as estratégias de diagnóstico utilizadas continuam a ser baseadas na identificação de algumas moléculas, muitas vezes inespecíficas de determinada situação. Urge, pois, desenvolver estratégias que permitam avaliar o cenário molecular completo. O SalivaPrint é uma dessas estratégias. Contudo, para que esta abordagem possa ser utilizada no diagnóstico há que padronizar os perfis obtidos e identificar os fatores que afetam este perfil, tais como a idade, o género e a condição oral dos indivíduos. Nesse sentido, este trabalho tem como objetivos: 1. Verificar se há variação do SalivaPrint com a idade e com o género; 2. Verificar se há variação do SalivaPrint com a saúde oral; 3. Verificar se há relação dos parâmetros físico-químicos da saliva com os ín- 